\title{
LA FORMACIÓN UNIVERSITARIA: LAS TRANSFORMACIONES ACADÉMICAS DE LOS ESTUDIANTES EN UNA SOCIEDAD HOSTIL
}

\author{
UNIVERSITY EDUCATION: ACADEMIC TRANSFORMATIONS OF \\ THE STUDENTS IN A HOSTILE SOCIETY
}

\section{FORMAÇÃO UNIVERSITÁRIA: TRANSFORMAÇÕES ACADÊMI- CAS DE ALUNOS EM UMA SOCIEDADE HOSTIL}

\section{Diego Mauricio BarragáN ${ }^{1}$}

Resumen El artículo explora las transformaciones académicas destacadas por los estudiantes durante su formación universitaria en una sociedad hostil. Se utilizó metodología cualitativa, tomando testimonios, observaciones y conversaciones, donde los jóvenes establecen lo importante de su experiencia estudiantil. La indagación se centró en el proceso de formación, las competencias intelectuales, el esfuerzo individual y las relaciones de los estudiantes con los profesores. A pesar de las críticas constantes a la universidad, en especial a la universidad pública, participar del proceso de formación ofrece a los estudiantes una ventana al mundo distinta a quienes están fuera de la educación superior, que pueden ser grupos de origen o con quienes comparten su cotidianidad; su paso por la universidad se proponen como una alternativa que le ofrece a los estudiantes elementos para entender cómo desarrollar su formación, encontrarse a sí mismo e interpretar y actuar sobre los sucesos de su realidad inmediata.

Palabras clave: Experiencia estudiantil; Formación universitaria; Transformaciones ACADÉMICAS Y SOCIEDAD HOSTIL.

Abstract The article explores the academic transformations highlighted by the students during their university education in a hostile society. Qualitative methodology was used, taking testimonies, observations and conversations where young people establish the importance of their student experience. The inquiry focused on the training process, the intellectual competencies, the individual effort and the students' relationships with the teachers. Despite constant criticism of the university, especially the public university, participating

\footnotetext{
Universidad Externado de Colombia.
} 
in the training process offers students a window into the world other than those who are outside higher education, who may be groups of origin or with whom they share their daily life; his passing through the university are proposed as an alternative that offers students elements to understand how to develop their training, find himself and interpret and act on the events of their immediate reality.

Key-words: Students experience; University education; Academics transformations and hostile society.

Resumo $\mathrm{O}$ artigo explora as transformações acadêmicas destacadas pelos alunos durante a sua formação universitária em uma sociedade hostil. Metodologia qualitativa foi utilizada, tendo testemunhos, observações e conversas em que os jovens estabelecem o quão importante é a sua experiência de estudante. A investigação incidiu sobre o processo de formação, habilidades intelectuais, esforço individual e relações dos alunos com os professores. Apesar da crítica constante da universidade, especialmente a universidade pública, parte do processo de formação dá aos alunos uma janela para um mundo diferente para aqueles que estão fora do ensino superior, que podem ser grupos de origem ou com quem eles compartilham suas vidas diárias; seu tempo na universidade é proposto como uma alternativa que oferece aos estudantes compreender como elementos para desenvolver a sua formação, encontrar-se, interpretar e agir acerca dos acontecimentos de sua realidade imediata.

Palavras-chave: Experiência do estudante; FormaÇão universitária; Transformações ACADÊMICAS E SOCIEDADE HOSTIL.

\section{INTRODUCCIÓN}

La formación se toma como los vínculos que establecen los estudiantes a lo largo de su proceso educativo, donde encuentran parámetros establecidos que ellos pueden seguir como referentes para sus actuaciones y sus relaciones con otros. Las redes de personas en la universidad, su presencia, su permanencia, sus logros, sus ámbitos de acción, se toman como lugares para tejer lazos entre personas con intereses similares, o medios para acercarse a ideas o actividades. Estos procesos generan en los estudiantes parámetros de solidaridad y cohesión, los hace sentirse miembros de algo, de un cuerpo que les posibilita identificarse y, en ocasiones, desarrollar actuaciones colectivas; les permite participar en un acumulado social de conocimiento y las prácticas derivadas. Significa pertenecer a grupos de personas que se vinculan para desarrollar propósitos comunes (ELÍAS, 2011, p. 15). Las lógicas de las redes establecen las prioridades a los miembros y las acciones eficaces para instaurarse o mantenerse. En instituciones donde no existen parámetros de actuación claros, las redes informales expanden sus lazos e instauran sus prácticas (BOURDIEU, 2013, p. 180).

El tránsito por la universidad va ofreciendo un panorama diferente a las personas, Carolina (estudiante de Licenciatura en Lengua Castellana) sostiene: 
...Yo creo que esos imaginarios van cambiando, se va clarificando un poco qué fue lo que decimos estudiar y claro, creo que cambia muchísimo el contexto, en la universidad aprender a conocer los espacios y en cuanto a la gente, conocer nuevos maestros, nuevos compañeros de clase, todo eso creo que sí implica muchas dinámicas.

En el desarrollo de la formación, las personas tienen acercamientos con el saber, las dinámicas institucionales, y, en especial, las comunidades profesionales o académicas; estas últimas son quienes las reciben y crean los espacios donde van a estar, los grupos tienen características particulares que establecen sus relaciones con el saber, en su interior, con el ámbito profesional y con diferentes instancias o personas del contexto (BECKER, et al., 1992). Son grupos o comunidades particulares que dejan su huella en los individuos (ELÍAS, 2000, p. 197). Se trata de procesos de socialización que no se reducen al rendimiento académico, lo que hacen es formar y transformar a las personas (DUBET, 2011, p. 64); son vínculos generados en la interacción dentro de los procesos, en este ámbito, los senderos para desarrollar su trayectoria son elegidos por las personas (ELÍAS, 1991, p. 22). Son observadores y son partícipes, son agentes y son productos, la doble condición los ubica en un limbo. A pesar de que son decisiones o senderos individuales, hacer parte de una comunidad les establece marcos para pensar, actuar (GOFFMAN, 2006, p. 35), sentir y observar. Es una interdependencia entre las dinámicas de las comunidades y los senderos que deciden o asumen las personas. Vincularse a estas comunidades transforma (ELÍAS, 2000, p. 198), en cierta medida, las experiencias de los estudiantes (FRENCH, 1997).

El artículo explora las transformaciones académicas destacadas por los estudiantes durante su formación en una sociedad caracterizada por la desigualdad y el conflicto. Los protagonistas se encuentran al final de su formación, ${ }^{2}$ tienen visiones, testimonios de su pasado y su presente, señalan lo que consideran importante y los vínculos que paulatinamente construyeron a lo largo del proceso; allí se evidencian las transformaciones. Las partes que lo componen son: a) su concepción y participación en el proceso educativo; b) las capacidades intelectuales que han identificado; c) el reconocimiento del esfuerzo individual, y d) los encuentros con los profesores. Metodológicamente, la investigación tiene un enfoque cualitativo, donde se trataron de utilizar herramientas que pudieran manifestar las experiencias significativas de los estudiantes (GOFFMAN, 2006: 17). En el trabajo de campo se aplicaron 17 entrevistas en profundidad, 9 grupos de discusión y observación participante, en total participaron 70 estudiantes de séptimo a décimo semestre, fue un número igual de hombres y mujeres y el rango de edad se encontraba entre 21 y 24 años, que pertenecían, salvo Filosofía, a los programas mayor tradición o de mayor número de estudiantes en las dos universidades donde se aplicaron los instrumentos, siguiendo la división características de la educación superior en Colombia, y de algunos países de América Latina, la división

2 El artículo es parte de la tesis doctoral de Diego Barragán titulada La experiencia estudiantil en una sociedad hostil. Una aproximación a los estudiantes universitarios de Ibagué (2012), desarrollada en el Doctorado en Ciencias Sociales de la Universidad de Buenos Aires y sustentada en 2017. 
entre la universidad pública, la Universidad de Tolima, y la universidad privada, la Universidad de Ibagué; ${ }^{3}$ en la ciudad de Ibagué en el centro de Colombia durante el año 2012.

\title{
1. El PROCESO DE FORMACIÓN
}

Los jóvenes entran a la universidad sin conocer o sin dimensionar lo que quieren o van a hacer; la mayoría se dan cuenta de ello cuando están en medio del proceso (DUBET, 2005, p. 38). En los primeros años de estudio, deben desarrollar una disciplina personal que les permita soportar los desafíos y una afiliación institucional para su permanencia en la universidad (MALINOWSKI, 2008); es un proceso de adoctrinamiento que desarrolla cada comunidad (ACOSTA, et al., 2011, p. 262). Marín (estudiante de Filosofía de la universidad privada y egresado de Licenciatura en Ciencias Sociales de la universidad pública), describe las transformaciones que él observa en los estudiantes:

\begin{abstract}
Al volverlo a ver (al estudiante) por allá en séptimo u octavo semestre y el cambio es radical, son mucho más disciplinados y ordenados en el aula de clase, a medida que va pasando el tiempo se adoctrinan y todo eso los va puliendo, es una exigencia que me parece muy lícita de la vida universitaria. Es como tener un tipo de ordenamiento y de disciplina para con el otro, para escucharlos, sino que en los primeros semestres es una cosa muy poco dada, y tiene que haber una relación muy lúdica con el conocimiento; hay uno que está cantando, el otro se está riendo, por eso la gente se aburre.
\end{abstract}

Un elemento que deben asumir los estudiantes, mencionado reiteradamente, es la responsabilidad, tanto con sus estudios como consigo mismos. Aprenden a combinar las reglas del juego con su desempeño (GUEVARA, 2009), asumiendo la responsabilidad como eje central del proceso. Andrea (estudiante de Comunicación Social), afirma:

\footnotetext{
... Mi vida sí ha cambiado... la disciplina cambia, ya es una responsabilidad tuya si quieres ir a clase, porque si no vas, pues pierdes, pero en la consciencia te queda: bueno, perdí pero por qué, porque no hice nada... tengo que hacer lo que sea, lo otro es que voy a tener nuevos amigos que se van a desarrollar en distintos ámbitos, entonces, toca llevar una responsabilidad mucho mayor.
}

La transformación la ilustra participantes del grupo de discusión en Licenciatura en Lengua Castellana, respecto a las personas que no aceptaron las condiciones y las dinámicas del espacio académico:

3 En la Universidad del Tolima los programas fueron: Administración de Empresas, Licencia en Lengua Castellana, Licenciatura en Ciencias Naturales, Biología y Economía. En la Universidad de Ibagué: Contaduría Pública, Filosofía, Comunicación Social, Ingeniería Industrial, Psicología y Arquitectura. 
[Entrevistado] Incluso hay quejas de los mismos docentes, generalmente se dejan materiales, libros para leer, el docente entra y dice, vamos a ver qué leyeron, quién leyó, por ahí dos o tres levantan la mano, técnicamente hablando en la medida que van avanzando los semestres se van reduciendo los grupos.

[Entrevistada] A mí siempre me gusta poner el ejemplo desde nosotros, nosotros entramos 56 estudiantes para el primer semestre y quedamos como 12. (...) con la mayoría uno conversa con ellos en el salón de clase, y se dice, él está acá porque realmente tiene las bases y el mérito para estar en séptimo semestre.

Es un proceso gradual que inicia en los primeros semestres, casi en el primer año (BENAVIDES et al., 2014) los estudiantes deben equilibrar sus proyectos con los lineamientos institucionales en dos sentidos; por un lado, en la formación intelectual, nuevos contenidos, nuevas materiales, nuevos conocimientos y, por otro lado, la inserción a un nuevo espacio, con lógicas distintas (GÓMEZ \& ÁLZATE, 2010), el individuo debe trazar su camino. Aquellos que no soportan las dinámicas de las comunidades, una vez que se encuentran al frente de los programas y las exigencias institucionales, deben salir; quienes se quedan, sufren una transformación orientada a las exigencias que proponen las instituciones y las comunidades (ROLDAN, 2014).

La deserción no se puede limitar a las responsabilidades de las personas, a pesar que generalmente se cargan en sus hombros. El Ministerio de Educación Nacional, por medio del Sistema Para la Prevención de la Deserción en Educación Superior (SPADIES), presenta el comportamiento de la deserción de 1998 a 2012 en Colombia. Toma la variable de ingresos de los estudiantes o la familia por Salario Mínimo Legal Vigente (S.M.L.V); un S.M.L.V corresponde a $\$ 644.350$ pesos colombianos (215 dólares). La escala de medición va desde 1 a 15 S.M.L.V. Para la ilustración se toman:

\begin{tabular}{|l|l|l|l|l|}
\hline $\begin{array}{l}\text { Angresos } \\
\text { salariales }\end{array}$ & $\mathbf{1 9 9 8 - 1}$ & $\mathbf{2 0 0 3 - 1}$ & $\mathbf{2 0 0 6 - 2}$ & $\mathbf{2 0 1 1 - 2}$ \\
\hline $\mathbf{1}$ SMLV a 315 US & & & & \\
\hline 7 SMLV a 2208 US & $24,26 \%$ & $55.50 \%$ & $59.38 \%$ & $62.19 \%$ \\
\hline $\mathbf{1 5}$ SMLV a 4733 US & $16,45 \%$ & $44,63 \%$ & $49,24 \%$ & $45,06 \%$ \\
\hline
\end{tabular}

Tabla 1: Medición de deserción en Colombia de acuerdo con los ingresos.

Fuente: (SPADIES, 2012). ${ }^{4}$

4 Es necesario señalar que las mediciones sobre deserción se presentan en forma bruta o por cohortes, las diferencias entre una y otra son significativas; pero, no son claras las metodologías utilizadas en la construcción de las variables y las mediciones. Por cohortes ilustran un poco mejor, por ello se utilizan (BARRAGÁN \& PATIÑO, 2013, p. 56). 
Desde la década de 1990, la deserción ha mostrado un aumento gradual y constante, sin diferencias significativas entre universidades públicas y privadas o personas con pocos o muchos ingresos; es una característica estructural del sistema universitario colombiano y centro de preocupación para las universidades y el Estado.

Adicional a la deserción, algo que normalmente se oculta o se desconoce es que en la universidad el proceso de formación se encuentra invadido de la cultura juvenil (DUBET, 2011, p. 68). No se puede segmentar y hacer una incisión entre las labores académicas y lo que viven los jóvenes. Los procesos de exigencia, de adoctrinamiento llegan hasta un lugar, de acuerdo a las condiciones de los estudiantes; participantes del grupo de discusión de Licenciatura en Ciencias Naturales sostienen que llega un momento el cual:

... Les falta motivación, y creo que uno necesita de algo para que lo motive para continuar con lo que uno quiere, porque a veces se le presentan inconvenientes en la vida que lo ponen a uno a dudar de las cosas, entonces, uno necesita de una motivación para continuar.

$\mathrm{Al}$ respecto, en medio de la entrevista con Howard (estudiante de Ingeniería Industrial), indica cómo lo afecta la falta de motivación y comienza a mezclar problemas personales con problemas del entorno:

Todo eso de manejar problemas es muy "maluco", tras de que le exigen a usted en la universidad y manejar los problemas de la casa y sobrellevarlos, muchas veces son difíciles de llevar y muchas veces generan problemas, se desbalancea todo y así no rinde nada. Yo he tenido también problemas, por ejemplo, el cambio económico de mi papá también me afectó mucho,... mis padres se separaron, entonces, a pesar de que soy el mayor de la familia me afectó mucho. Ya uno busca como maneras de evitar ese problema y se entretiene en otras cosas, trata uno como de meterse en el tema del estudio para olvidar, pero siempre lo afecta a uno, porque es un ámbito que usted lo encuentra inherente en toda la familia y todo eso afecta... la vida en pareja, la vida universitaria, el rendimiento académico, y sobre todo llegar uno todos los días a enfrentarse al problema y eso lo afecta a uno muchísimo.

Muchas cosas afectan simultáneamente el rendimiento académico, problemas personales, familiares, económicos. No solo se trata de observar cómo se desenvuelven en el rendimiento académico (PAOLONI, 2011). Sino cómo la comunidad específica de la universidad, directa o indirectamente, les ofrece formas de afrontar sus problemas. Se tejen redes de interdependencias.

El proceso de formación vincula a las personas a las dinámicas institucionales, avanzar implica que asuman la disciplina y las normas establecidas en la institución y caminos para sortear los obstáculos o las dificultades. Se encuentran con varias comunidades dentro del proceso, se destacan las relacionadas con su formación disciplinal y los grupos con los cuales generan vínculos afectivos o ideológicos. Además, los jóvenes se involucran en múltiples actividades que deben combinar con sus estudios, en especial, trabajar, que 
los obliga a repartir su tiempo (VÁZQUEZ, 2009), deben mantener un equilibrio entre las actividades, sosteniendo un esfuerzo prolongado durante su formación. Desde luego, los estudiantes encuentran senderos para desarrollar su proceso.

\section{LAS CAPACIDADES INTELECTUALES}

Las capacidades intelectuales son las dimensiones que intervienen durante la formación, a nivel universitario, donde las personas establecen herramientas que les permiten comprender su mundo, reconocer quiénes son y desenvolverse en los procesos educativos. La dimensión destacada es la relación de la persona con el conocimiento (GUEVARA, 2009, p. 221); como lo mencionaba Chávez, (2008), en correspondencia a su origen educativo y social, las personas han desarrollado herramientas que les permiten desenvolverse en su medio cuando se enfrentan a lógicas distintas, a contenidos nuevos, a formas de observar y actuar en la realidad; se ponen a prueba, pueden enfrentar las nuevas circunstancias, abandonarlas o tratar de superar la obligación sin vincularlo a su acumulado de saber. Es decir, los estudiantes toman las capacidades intelectuales, las ponen a prueba y las aplican para sortear la situación a la que se enfrentan.

La primera relación de las capacidades intelectuales que se encontró fue entre profesores y estudiantes. Los profesores establecen dinámicas y establecen las condiciones de evaluación del desempeño de los estudiantes, dicen quienes hacen las cosas bien y quiénes no. Los estudiantes se orientan a superar los obstáculos, a aprovechar oportunidades y a jugar con las relaciones establecidas (NOBILE \& ARROYO, 2015). Las herramientas que tienen a su disposición son resultado de un proceso social, algunas veces lejanos de los espacios académicos, pero se interrelacionan, orientan o determinan los procesos académicos.

\footnotetext{
... Los alumnos poseen una serie de características materiales que son analíticamente independientes de las percepciones de los maestros. No obstante, dichas características son traducidas con cierta significación por parte del docente en interacción con sus alumnos... Este conocimiento que tiene el maestro respecto de sus alumnos es entonces en parte construido por él, en base a sus propios esquemas de apreciación y valoración diferencial. Por lo tanto, al mismo tiempo que el maestro conoce a sus alumnos, los clasifica o categoriza (KAPLAN, 1994, p. 25).
}

Las evaluaciones, las críticas, las actividades, las reflexiones de los profesores orientan la formación de los estudiantes (DUBET, 2005, p. 38); tal vez, su tarea es desarrollar una ruta que le permita avanzar sin tener obstáculos, aprovechando los espacios y las interacciones que brinda la universidad. En este ámbito, las capacidades son las herramientas básicas, son las condiciones que tienen las personas para aprender o cultivar distintos campos del saber y desempeñarse en ámbitos de acción en el desarrollo del proceso de formación. Participantes del grupo de discusión en Comunicación Social sostienen: 
Para nosotros la capacidad intelectual, es... no crear unos estándares, pero sí tener unos objetivos claros, como que esa persona pueda desarrollar sus capacidades a partir de lo que les exigen en las universidades.

El camino usualmente transitado para la definición de las capacidades intelectuales de los estudiantes es la cuantificación. Según estudiantes del grupo de discusión en Licenciatura Ciencias Naturales: “...las capacidades intelectuales se ven reflejadas en la nota, esa es como la valoración que le dan". Continúan con una reflexión sobre cómo la nota se ha convertido en el centro de preocupación, no son las capacidades que se demuestran, es cómo se representan en la planilla de calificaciones del profesor:

Además de que nosotros hemos venido como creciendo... con la mentalidad de
preocuparnos por el número o por una letra, más allá de si aprendimos y si ad-
quirimos el conocimiento que era, da igual muchas veces, porque con tal de ver
un 3 o un 3.5 o la nota que sea, siempre y cuando no salga la que es rojita, uno
queda satisfecho. Es como la mentalidad con la que venimos creciendo, por eso
es muy difícil también que se identifiquen todas esas capacidades individuales,
porque nos preocupamos más es por pasar, sea como sea, entonces, es difícil.

Aquellos que pertenecen a las comunidades de ciencias económicas: Administración de Empresas, Economía y Contaduría Pública encuentran sus referentes fuera de la universidad, en el "mundo real", en las empresas privadas vinculadas a los mercados internacionales. Para los profesores, y la comunidad en general, es necesario establecer los vínculos con el sector productivo; sin embargo, esto representa un problema en contextos económicos donde se encuentran pocas empresas y pocos empresarios. La mayoría de las actividades económicas se refiere a la subsistencia, encuentra una brecha entre la formación y "el mundo real". Miembros del grupo de discusión en Administración de Empresas lo sintetizan:

Simplemente estamos aquí como por unas notas y la mayoría se preocupa por pasar las materias y no se preocupa por aprender más, nos quedamos solamente con lo que vemos en el salón, entonces, no nos estamos apropiando en sí de la carrera como tal.

Esta tendencia hace que toda la comunidad académica, los estudiantes y profesores, dejen al margen o no valoren las capacidades. Estudiantes del grupo de discusión en Economía sostienen:

...no creo que se valore como es necesario dentro de la comunidad académica, las capacidades intelectuales en este momento no se les da la importancia que necesita por parte del estudiantado, por la conformación de la comunidad educativa, el reglamento estudiantil... pues no sustenta ese tipo de cosas.

Según ellos, no es solo el momento de la evaluación; es la dinámica general de la comunidad académica en la universidad, en la cual la valoración de las capacidades inte- 
lectuales de los estudiantes queda al margen y se trasladan a primer plano otro tipo de preocupaciones. Dentro del trabajo de campo, fueron constantemente mencionados los sistemas de calidad que se tienen en la educación superior colombiana. ${ }^{5}$ A pesar de la importancia de los exámenes y las notas, los estudiantes reconocen que durante el proceso de formación se exagera la trascendencia de las mismas, pero ello depende en gran medida de los profesores. Participantes del grupo de discusión en Licenciatura en Lengua Castellana dicen:

\begin{abstract}
...en nuestras capacidades intelectuales ellos (los profesores) ven es un número, entonces, solamente es una evaluación la que nos rige. En esa capacidad en donde no hay un desarrollo, un aprendizaje, sino que usted simboliza un número como lo dice John, pero más allá de eso qué hay, ¿tenemos fundamentos?, ¿algún proceso de desarrollo?, o simplemente de los números no se pasa uno porque no les interesa tener un proceso de aprendizaje a través de los semestres o simplemente dan la cátedra por darla. Entonces, pienso que no depende solamente de nuestro esfuerzo obtener nuestro mérito como estudiantes, sino también entraría en jugar el mérito del docente, ¿qué capacidades tiene él para exigir o no?, y ¿cómo lo demuestra a través de esa evaluación?, ¿por qué regularmente se hacen evaluaciones para todos los profesores?, hacen diferentes preguntas y una de ellas es: si las evaluaciones que nos brindan a nosotros en nuestros trabajos, si tienen una retroalimentación después de que se realizan, generalmente no se hace, nos entrega la nota y hasta ahí se acabó la evaluación. Entonces, no se tiene un proceso continuo porque es la evaluación la que simboliza todo acá en la academia, la nota es la que determina si somos de una expresión intelectual o no.
\end{abstract}

La necesidad de interacción entre los estudiantes y los docentes desborda la nota. La evaluación y la relación con el estudiante deberían profundizar y no estancarse en las mediciones dentro del proceso. Según los miembros del grupo de discusión en Arquitectura:

\begin{abstract}
...claro, con el tema que a mi edad no quiero hacer nada, pero también llego a una clase en donde hay un profesor deficiente, en donde hay un profesor que a mí no me exija, que yo vea que llega un profesor y que llegue a preparar la clase a última hora, entonces, no hago nada y simplemente qué pienso, la que pierdo al final soy yo y al profesor al igual no le importa...
\end{abstract}

Estudiantes del grupo de discusión de Licenciatura en Lengua Castellana sostienen que el profesor se orienta a cumplir con los deberes que le son asignados, y por los cuales está siendo evaluado:

5 Las personas que participaron en el estudio estaban ad portas de presentar el examen Saber-Pro (examen estatal que mide las competencias educativas, existen en primaria y secundaria). ¿Cuál es la preocupación? Los resultados del examen sirven para clasificar a los estudiantes y a las carreras, se utilizan para acceder a posgrados y, cada vez más frecuente, para acceder a empleos; los resultados de los test estatales inciden en su futuro. 
... Creo que ese proceso de evaluación se hace solamente como un protocolo, en donde no trasciende al analizar los resultados porque, nosotros como estudiantes nos comunicamos y coincidimos en que muchos profesores no deberían de tener el cargo que tienen, en la evaluación cada uno demuestra y representa lo que el profesor simboliza para ver cosas no solamente personales, sino más bien como la labor docente y de ahí esa evaluación nunca trasciende.

Con un ingrediente adicional que interviene en la relación de los estudiantes y los profesores, que lo mencionan participantes del grupo de discusión de Licenciatura en Ciencias Naturales:

... pienso... que al profesor le debe de quedar como difícil, manejando mínimo 20 o 25 estudiantes y como máximo 50, entonces por ejemplo, si se ven solamente dos veces por semana en las clases, entonces, él sabe que tiene que enseñarles ciertos temas a los estudiantes y que cuando salgan de esa clase tienen que haber aprendido a desarrollar algún tema o algo. Él va es explícitamente es a dictar su clase y a enseñar el tema y él tiene que saber quiénes aprendieron, de pronto no hay como ese tiempo o no va a tener la oportunidad de examinar a cada estudiante, sin saber qué hay más allá de la materia.

En las universidades públicas el límite es de 50 personas por salón, en las privadas no existen límites, pueden ir de 20 a 100, dependiendo del número de inscritos y la capacidad de las instalaciones. Algunos profesores dictan en una universidad 5 o 6 materias y si trabajan en dos puede llegar a dictar en conjunto 8 o 10 asignaturas. En un ejercicio numérico simple, con 50 estudiantes por grupo y asumiendo 9 asignaturas, tendría a la semana relación con 450 personas. ${ }^{6}$ Esto plantea algunas dudas: ¿cómo el profesor asume ésta situación? ¿Cómo evalúan un número alto de estudiantes? Participantes del grupo de discusión en Licenciatura en Lengua Castellana ilustran la situación en una carrera donde la producción de textos es fundamental e implica tiempo y esfuerzo en la evaluación:

Digamos que esas capacidades intelectuales acá son medibles en las dos ver-
tientes: la de cuantificar y cualificar, en esos términos por ejemplo, hay gente
que en el esfuerzo como tal para escribir un texto, se hacen ejercicios y cumplen
con determinadas características... Uno se da cuenta que hay docentes que mi-
ran caras y no textos, uno ve al profesor con el arrume de libros, de trabajos y se

6 Este ejercicio es tomado de las conversaciones desarrolladas con profesores durante el trabajo de campo, en algunos casos tenían un número mayor de estudiantes. La razón para tomar un número elevado de asignaturas se explica por los salarios bajos de los profesores. Para obtener ingresos necesarios para un nivel de vida básico deben trabajar por lo menos en dos lugares; algunos que tienen obligaciones económicas (créditos a largo plazo como vivienda o vehículo, tarjetas de crédito o consumos permanentes relativamente altos) deben trabajar en varios lugares a la vez. Para hacer un ejercicio simple, un profesor que trabaje en dos lugares tiempo completo, siguiendo la legislación colombiana donde el horario de trabajo son 8 horas, debe trabajar 16 horas diarias, es decir, 80 horas a la semana, en ocasiones incluyendo sábado y domingo. Este tiempo laboral excesivo afecta a los profesores, su desgaste y su esfuerzo son altos, algunos con serios problemas de salud. Por supuesto, también afecta a los estudiantes, pues no es lo mismo un maestro que puede dedicarse con detalle a una clase que aquel que debe preparar 9 clases diferentes. 
pone a mirar caras, ¡ah! a este pongámosle cinco, ya uno tiene cara de número, yo por ejemplo tengo cara de cuatro con cinco, es un ejemplo, porque yo tengo cara es de tres. Entonces, como que ya uno está catalogado, y no es que esté siguiendo la voz constante de la universidad de que todos somos un número más, la cuestión es que cuando un profesor lo evalúe a uno, que le haga el numerito, pero que también le haga una crítica del texto.

Siguiendo con el testimonio, generalmente aparecen hitos que van en contra de lo que ellos ejemplifican; por lo general, estas iniciativas que se alejan del modelo basado en la nota, dependen absolutamente de las capacidades y condiciones de los maestros, y representan para los estudiantes puntos de referencia para su formación, donde las capacidades intelectuales se evidencian:

... Se parece a un docente que tuvimos que se llama Carlos... él le ponía a uno la nota y cada párrafo le corregía haciéndole otro texto, eso era escriba y escriba y le decía: consulte a tal, eso es un ejercicio muy, muy bueno, en el cual uno reflexiona y sabe si realmente está o no está cumpliendo con ese compromiso académico que le dicta la universidad y si se está o no formando académicamente...

Es una forma de enfrentar el lenguaje que se utiliza, las herramientas que se desarrollan en el proceso, son los momentos donde las capacidades se afirman. Continúa el estudiante del grupo de discusión en Lengua Castellana:

... Póngase serio viejo, porque aquí no venimos a hablar de chistes, aquí estamos es dentro de la academia y tenemos que hablarnos en términos teóricos, tenemos que hablar en medio de un diálogo conceptual, en donde apliquemos supuestamente lo aprendido, entonces, son circunstancias y una carencia dentro de la academia...

A pesar de las condiciones adversas, generales, hay muchos profesores que dejan huellas en los procesos de formación. Ellos permiten a los estudiantes apropiarse de su proceso de aprendizaje, incorporar actividades de desarrollo y los incitan a asumir para sí las herramientas que se utilizan en los campos del saber; es decir, son maestros que amplían e incentivan las capacidades intelectuales de los estudiantes.

Sin embargo, para que el joven incorpore todo lo que un buen maestro le brinda, requiere tener disposición para el aprendizaje y esfuerzo. Es necesario desarrollar un equilibrio dentro de un proceso, donde las personas deben someterse a pruebas de diferente orden. Miembros del grupo de discusión en Arquitectura ${ }^{7}$ sostienen al respecto:

7 Diferente de lo mencionado para la Licenciatura en Lengua Castellana, en Arquitectura los estudiantes deben desarrollar proyectos de semestre que son elaboraciones a escala de construcciones o edificaciones, que implican esfuerzos desde diferentes ámbitos del saber. Contaban que antes de la entrega del proyecto, duraban varios días sin dormir, necesitaban recursos económicos, espacios para desarrollarlos, etc. 
... Pues yo tengo un compañero que es muy inteligente pero que no es constante o no es una persona juiciosa. Uno no alcanza el mérito en esta carrera solamente por ser el más inteligente o solamente por ser juicioso, uno necesita de las dos cosas, porque mucho trabajo no significa un trabajo bueno, ni poco trabajo le resulta chévere, las dos cosas tienen que ser acompañadas... tenemos el caso de un compañero, él es muy bueno, tiene muy buenas ideas, pero el tema es que a la hora de hacer, no hace nada. Para mí todo ese proceso intelectual se va para la caneca, porque no es capaz de llevarlo a la realidad.

Las dos dimensiones se integran en la realización de los proyectos académicos, se encuentran en una interrelación que les ayuda a soportar el proceso de formación y las exigencias. Son capacidades que se tienen que desarrollar, de aprender e incorporar en la formación. Pero, no las únicas.

Según Yolanda (estudiante de Licenciatura en Ciencias Naturales), pasar por la universidad abre la puerta para comprender lo que acontece en la vida cotidiana, ofrece miradas; son capacidades que se desarrollan en interacción con personas lejanas de la universidad:

El estudio le da a uno como un desenvolvimiento en muchos campos, el estudio no es solamente adquirir conocimiento sobre un tema conciso, sino aprender a desenvolverse... es porque me demostré que no es lo mismo estar uno sin estudio, que estar uno estudiando, y eso se lo demuestra uno en cualquier parte, en una reunión social, a donde uno vaya, llega a cualquier parte y tiene que decir algo de lo que usted hace acá. Es muy bonito decirlo y más cuando usted se lo ha ganado a pulso... por ejemplo, le preguntan a uno ¿usted qué hace?, o están en un programa por televisión y que uno pueda opinar, eso es chévere, usted sí sabe y no es que yo sepa mucho y no es que tenga que saber el significado de tal palabra, no, es que uno aprende a relacionar eso con la vida cotidiana, lo que uno sabe con la vida cotidiana y le puede dar un significado a las cosas.

Indudablemente, cuando se menciona a los estudiantes, el rol que ocupan las capacidades intelectuales en el proceso de formación aparece en primera instancia y se materializa en el sistema a través de una nota. En la manera en que se establecen las relaciones entre profesores y estudiantes. Pero, luego comienzan a salir las transformaciones que ellos han sufrido y las han incorporado a sus actuaciones, desde luego las capacidades para enfrentar los procesos académicos desde la constancia y la capacidad de aprendizaje; desde las cercanías que han tenido con personas que representan un ejercicio intelectual constante y que enriquecen su formación, desde las posibilidades y los espacios que la universidad les ha abierto. Con un ingrediente adicional, sus relaciones con las personas lejanas a la universidad se modifican, por ser parte de la comunidad universitaria, pero también por hacer interpretaciones, tener las herramientas que les permite entender la realidad donde viven, el mundo universitario les ofrecen a las personas visiones diferentes del contexto de vida. 


\title{
3. El ESFUERZO INDIVIDUAL
}

La magnitud y la orientación del esfuerzo individual dependen de los contextos donde se desarrolla, más cuando son procesos educativos. Existen tradiciones donde las capacidades de las personas y los méritos dependen del esfuerzo individual (MÉNDEZ, 2010; YOUNG, 1963). Desde hace un tiempo, algunos discursos que permean la educación han descontextualizado el esfuerzo, han olvidado que hace parte de las interacciones desarrolladas durante un proceso y, sobre todo, han puesto toda la carga sobre el individuo, él es el responsable (ELÍAS, 2000). Esta visión se ha intensificado desde las reformas estatales de finales de los 80 donde el bienestar y el futuro se desligaban de las obligaciones del Estado, a pesar de los aumentos de la carga impositiva, para pasar a la responsabilidad del propio individuo. Con un ingrediente adicional, en un contexto de desigualdad y conflicto, el esfuerzo del individuo se convierte en una esperanza, un referente de actuación en las personas repetido y validado en la cotidianidad. Alberto (estudiante de Administración de Empresas) ilustra esta posición:

...siempre he dicho que las opciones uno las crea y existen en todo lugar, están ahí, hay muchas personas que tienen formación y están desempleadas, eso depende de la calidad de vida que uno quisiera llevar y de la forma como uno se enfoca, sencillamente.

Este punto de vista se refuerza con las declaraciones de Eduardo (estudiante de Administración de Empresas):

\begin{abstract}
Bueno, en los estudiantes en sí yo tengo una concepción y es que cada persona es el resultado de lo que quiere ser, independientemente de que se desarrolle en un ambiente violento o no, que puede generar algún tipo de repercusiones es cierto. Pero reitero, una persona es el resultado de lo que quiere ser, puede en ocasiones afectar en el sentido o en factores como el desempleo y la falta de oportunidades, la violencia... digamos que esos factores de desempleo y la falta de oportunidades sí pueden llegar a trascender dentro de la formación como estudiante, mas no el hecho de la violencia porque cada persona adquiere y extrae del medio en que se rodea lo que en realidad quiere para ella.
\end{abstract}

La desconexión del contexto limita, establece la carga en los hombros de las personas (KAPLAN, 2008). Son muchas las voces que le reiteran a las personas que ellas son las responsables de lo que acontece en su cotidianidad. Se destacan las cargas asignadas a los jóvenes, según las cuales ellos son el futuro, los responsables del futuro y lo deben asumir. ${ }^{8}$ Los problemas surgen cuando se enfrentan a contextos donde son pocos los proyectos coherentes de futuro, la frustración o la decepción la asumen los jóvenes (GOFFMAN, 2013).

8 En los medios de comunicación se presentan gurús de la motivación y el esfuerzo. Desde luego, todo se carga al individuo. Cada año crece la producción y venta de materiales como videos, libros, páginas web, etc.; donde se presentan y se valida el discurso de los "líderes", del esfuerzo individual como factor esencial, todos ellos desconectados del contexto. 
... A pesar de la falta de recursos muchos estudiantes han logrado sus objetivos, hay compañeros que no tienen dinero para el transporte, la alimentación... por ese tipo de situaciones realizan un gran esfuerzo para venir a estudiar. Hay estudiantes que tienen mérito porque buscan aprender más de lo que se les enseña, no buscan la mediocridad sino mayor conocimiento, también hay mérito para los maestros que reconocen el esfuerzo de los estudiantes y les dan estímulos (Miembros del grupo de discusión en Economía).

Es clara la manera en la cual los jóvenes que adelantan un proceso de formación identifican el esfuerzo individual como el indicador o la unidad de éxito del proceso que vienen adelantando, un espacio donde gradualmente sufren transformaciones (YAIR, 2008, p. 99). Participantes en el grupo de discusión en Administración de Empresas sostienen:

A mí me parece bien en cuanto a lo que dice Andrés y a la larga o al final el esfuerzo se verá bien recompensado, siempre se va a ver bien retribuido, es importante ser uno exigente consigo mismo y ser uno disciplinado, por eso me parece que a uno le va a ir bien si uno se esfuerza, porque uno hace las cosas de corazón.

El grupo de discusión en Arquitectura, también aborda el tema:

El esfuerzo individual que tiene la carrera, pienso yo que es de uno mismo y que uno ha participado desde el principio hasta donde está ahora, entonces, uno reflexiona y dice: he sido estudiante, he sido vago, he estudiado mucho, sí o no.

Este indicador se utiliza como herramienta para determinar cuál fue "mi desempeño" en un proceso de formación, desde luego, la persona es la responsable, pero la diferencia radica en que se establecen los vínculos con el contexto. Las evaluaciones, las posiciones, los resultados dependen de las interdependencias con quienes hacen parte del proceso.

Los resultados dependen de cómo los estudiantes asimilen el proceso. Respecto a este punto, Lucero (estudiante de Contaduría Pública) afirma:

\footnotetext{
...un estudiante que entra... con las ganas de salir adelante, entra a estudiar lo que realmente le gusta, entonces, su rendimiento va a ser alto, porque sabe que si no es alto puede perder la oportunidad de estudio, te vas a esforzar más, por eso es que digo que eso va en la persona. En cambio, un estudiante que tiene absolutamente todo, que sus papás le dan todo, yo conozco estudiantes que tienden a ser muy dejados de la universidad y es porque siempre lo han tenido todo. Por eso es que digo que eso va en la persona y no en la universidad, el rendimiento va en la persona, si una persona quiere.
}

Existen opciones diferentes de asumir proceso de formación, algunos se esfuerzan, disfrutan lo que hacen, se sienten a gusto con lo que estudian, en sus carreras, sus espacios y tienen una proyección positiva para el futuro. Otros entran a la universidad por razones que no comparten, no les gusta lo que hacen, no lo disfrutan y establecen obstáculos para 
quienes asumen el proceso; así, no quieren esforzarse y deben terminar la universidad, su mente y sus expectativas se encuentran en otro lugar, pero saben que terminar la universidad les representa posibilidades. Participantes del grupo de discusión en Contaduría Pública ejemplifica esta posición:

Acá todo es fácil, lo que pasa es que si tú ves que hay una materia fácil de pasar, tú te arreglas así y pasas, inclusive con buenas notas, uno pasa con buenas notas y son materias que no te exigen nada y siempre vas a tener un cuatro con cinco. Ese es un obstáculo grande, uno se vuelve muy flojo, siempre que uno va a comenzar semestre, pregunta quién nos va a dar tal materia, no, que tal persona; ya pasé...

Personas que optan por este sendero hacen parte del día a día de las aulas de clase asumen su proceso de formación desde la comodidad y haciendo el menor esfuerzo posible; claro esto, con la complacencia de los profesores. Miembros del grupo de discusión de Administración de Empresas sostienen:

... pienso lo mismo, ha habido muchas falencias, hemos tenido muchos profesores que realmente producen es como lástima, ha habido materias que han sido un total relleno, que le están quitando el cupo a otra materia. Eso es un fallo grave, pero es lo mismo, uno siempre vive inconforme con las cosas y uno debe quejarse y la gente es como muy pasiva en ese sentido, como es una materia fácil, entonces, hagámosle y así todos en el paseo... Uno debe sacarle el jugo a lo que sea, así sea una materia mala, desafortunadamente y de acuerdo a mi experiencia personal, yo no lo hago, yo he desaprovechado muchas materias y yo me pongo a pensar, pero bueno, si yo sigo así, ¿qué clase de profesional voy a ser el día de mañana?, el hecho es aprovechar y aprovechar y realmente uno sí se queda callado, porque es que la mayoría de la gente lo hace.

A diferencia de lo que sostenía Marín (estudiante de Filosofía) (ver, 1. El proceso de formación) sobre cómo los estudiantes se van transformando en el desarrollo del proceso de formación, es decir, en los primeros semestres tienen hábitos del colegio que dificultan el desarrollo de actividades en la universidad, las formas de asumir el conocimiento y las maneras de comportamiento. Con el paso del tiempo en los últimos semestres, han adquirido dinámicas de la comunidad profesional, disciplina, orden y esfuerzo. Participantes del grupo de discusión en Contaduría Pública plateaban un proceso inverso entre el rendimiento académico y esfuerzo.

Pues, personalmente yo he vivido desde los primeros semestres, yo he sido como muy dedicado, juicioso, tuve buenas notas y pasaba los semestres. Pero ya este último, como que ya veo que simplemente es pasar, bacano ser uno bueno, demostrar ser el mejor, obtener buenas notas, pero también uno puede subsistir en la universidad y poder sacar el cartón manejando un perfil suave y fácil, sin tanto esfuerzo e ir pasando las materias. Últimamente los semestres lo he pasado así, suave, casi no me he esforzado y he ido pasando, voy nivelado... 
personalmente a mí me pasa, pues estoy trabajando, tengo las obligaciones del trabajo y a veces me cuesta llegar, ocho horas trabajando y luego venir acá a recibir clase (cuatro horas), entonces, se hace más difícil para hacer tareas, llevo todo el día trabajando, entonces, como que uno está muy cansado. Ya lo que uno piensa es en pasar la materia y ya no va a sobresalir.

Julia (estudiante de Contaduría Pública) hace una interpretación de esta posición:

Sí, se nota que algunos llegaron acá y quieren que se les diga, ustedes están pagando una matrícula súper alta, no, porque no se esfuerzan para que la nota sea buena. No se les ve un interés, yo no sé, deberían esforzarse más y como valorar esas cosas, porque vamos a salir profesionales y no vamos a salir con un cuento por ahí todo raro.

Es un poco contradictorio. Se tiene el ideal de esfuerzo en la universidad, el individuo asume un proceso que va a traer frutos en el futuro, pero existen estudiantes en el presente que lo "toman suave", que viven pensando o habitando otros espacios, donde la universidad y la formación ocupan lugares secundarios. El problema genera una tensión en la representación entre quienes asumen con seriedad la formación y quienes no, o les importa poco.

La forma de asumir el esfuerzo se evidencia en los trabajos en grupo. Es la responsabilidad que tienen los jóvenes con sí mismos, se manifiesta, interfiere o facilita la relación con las otras personas y el cumplimiento de sus obligaciones. Participantes en el grupo de discusión en Administración de Empresas planteaban:

...creo que la vida se trata es de portarse uno mismo, de retroalimentarse uno mismo a través de muchas cosas, pedirse uno y saber pedirle a los demás, no necesariamente tengo que quedarme con lo mío. Ahora, si yo estoy en contrario a muchas cosas porque si yo estoy mal y el otro tiene la solución con su saber, entonces, me acerco y pido colaboración. Obviamente sin tener en la mente algo a cambio, de todas maneras el esfuerzo personal de uno requiere de unas exigencias hacia uno mismo y a los demás para poder cumplir con las metas que uno tiene.

Participantes del grupo de discusión en Arquitectura, agregan un nuevo elemento:

Al igual, usted puede estar en grupos malos, porque cuando uno está con los buenos, es llevarle la idea al otro, igualmente yo tengo que demostrar que yo puedo ser igual a las demás personas, entonces, es como que el esfuerzo lo que lo marca a uno, que uno tiene que aprender...además que otro factor fundamental para el esfuerzo individual es la competencia, ¿por qué?, porque por lo menos yo estudio con unos compañeros que son muy buenos y que generan mucha competencia y simplemente uno dice que no puede uno llegar con cual quier bobada a la universidad. 
Las interacciones en los grupos establecen dinámicas de trabajo, de relaciones y de las posiciones que asumen los individuos. Se encuentran en un proceso educativo, donde deben estar en contacto permanente con los otros. Miembro del grupo de discusión en Licenciatura en Ciencias Naturales lo ilustra:

\begin{abstract}
Eso trata de ser contradictorio, a veces uno necesita del otro compañero, porque muchas veces uno es bueno para unas cosas y malo para otras... por lo general uno se colabora entre todos y siempre irá a haber alguien que haga las cosas, ya sea porque a veces las presta o algo así, yo creo que todos nos hemos aprovechado de eso.
\end{abstract}

Así comienzan a desarrollar lazos que les permiten sortear diferentes exigencias y obstáculos que se presentan en el proceso. Participar de una comunidad donde se establecen lazos, donde ellos van conquistando escaños en el proceso de formación, hace que los lazos y las relaciones con las personas se construyan, con quienes trabajan a gusto, con quiénes pueden aventurarse a exigencias altas y con quiénes no.

Esta idea se confirma según lo planteado por miembros del grupo de discusión en Ingeniería Industrial, ellos afirman:

Pues en realidad, uno habiendo escogido a las personas del grupo, desde que comienza la carrera, empieza como a conocer a las personas y las invita a formar un equipo en el que uno sabe que se entiende y que puede trabajar. Uno siempre que hace un grupo, ya sabe con quién trabajar y si uno conoce a alguien que no trabaja, uno no se va a hacer con él, porque es una nota y uno sabe que hay que trabajar y la responsabilidad no va caer solamente en una persona.

Estudiantes del grupo de discusión en Psicología describen las dinámicas:

... yo digo, eso también va en el alumno, a mí no me parece que porque es un trabajo en grupo, que no se les explota sus habilidades, a mí me parece que eso va en que si uno tiene un trabajo en grupo, cuando uno hace un trabajo bien y que le queda bonito y le va bien, es porque es un esfuerzo de todos, eso no es porque solamente uno hizo o no hizo, no, eso es un esfuerzo de todos. Sí hay trabajos que no son tan buenos, es porque el esfuerzo se ve de que hay gente que no trabaja o que no sé qué, es que uno se da cuenta, no que no quieran explotar sus capacidades y puede que la gente sea creativa, pero puede ser perezosa, porque es que hay grupos que son pequeños y uno se da cuenta porque a la gente le da pereza. Yo he tenido experiencias en cuanto a que hemos incluido a otras personas en el grupo, y la gente puede que sea creativa y puede saber cosas, pero la gente es perezosa, eso hay que aceptarlo, hasta a uno mismo le da pereza.

Una constante en los testimonios en cuanto al tema de los trabajos en grupo fue las reiteradas menciones a que muchas personas tienen capacidades, tienen posibilidades de desarrollar actividades productivas, pero no tienen la disposición, aquí se llaman "perezosas". Sin embargo, el trabajo en grupo hace parte del proceso de formación, en muchas 
carreras el trabajo en grupo es eje central, p.e. Arquitectura, Psicología o Biología. El nivel del esfuerzo establece la dinámica del trabajo en grupo. Es posible que todos trabajen y se retroalimenten; que algunos trabajen y otros los acompañen; que el trabajo es responsabilidad de una persona y los otros no hacen nada o que ninguno hace nada. Los integrantes del grupo de discusión en Administración de Empresas ilustran estas situaciones:

Pero igual nosotros ayudamos a que los demás compañeros pasen, es como saber que uno fue el que hizo el trabajo y el otro es el que se lleva la nota, es injusto que la persona que trabajó saque la misma nota que el que no trabajó, o de buenas porque sacó la nota más alta en un parcial.

Al esfuerzo individual se suma la inteligencia práctica (BOURDIEU, 2013, p. 142). Es la capacidad de ubicarse en un contexto y establecer las relaciones apropiadas con el esfuerzo que se realiza. Saben que están dentro de un proceso y que necesitan relaciones diferentes con algunas personas, lo que hacen es identificar cómo establecer apropiadamente esas relaciones en beneficio de su proceso de formación.

\section{LOS ENCUENTROS CON LOS PROFESORES}

Las relaciones entre profesores y estudiantes siempre han sido paradójicas. Soler (et al. 2009, p. 7) señalaba que las relaciones significativas eran entre estudiantes, entre sus pares, y en menor medida, con los profesores. Son encuentros de dos mundos distintos, estudiantes y profesores, cuyas distancias cada vez se amplían, se manifiestan en los conflictos que se viven cotidianamente en las aulas y en la universidad; reforzados por la pasividad, la indiferencia de los estudiantes, la autoridad gradualmente en decadencia y los problemas de comunicación que los profesores encarnan (GRAFF, 1995, p. 278). Muchas veces, el desempeño de los estudiantes depende de las relaciones que tejen con los profesores (NOBILE \& ARROYO, 2015), estas inciden de forma fundamental en el futuro académico de los jóvenes; desafortunadamente, incluyendo los prejuicios de los profesores (KAPLAN, 2008).

Una de las ilustraciones de mayor fuerza, con respecto a los lazos que se tejen, la hizo Yolanda (estudiante de Licenciatura en Ciencias Naturales), ella señala:

\footnotetext{
...aquí en el laboratorio se aprende mucho a conocer a las personas, porque es que compartimos mucho tiempo, nosotros nos volvemos una familia. Así como una experiencia rápida, hace poco tuve un problema de salud muy grave... los médicos me diagnosticaron que me había dado un paro cardiorrespiratorio durante 40 minutos, me operaron del corazón y estuve muy mal, estuve ausente casi un mes. Todos ellos fueron la familia que yo tuve, ellos eran los que iban y me visitaban todos los días, el director del laboratorio fue el que me apoyó en absolutamente todo, otro integrante del laboratorio también me apoyó muchísimo, estuvo conmigo en todo. Son de esas personas que se vuelven parte de la familia de uno, entonces, eso transciende un poquito al ámbito laboral...
} 
Las comunidades donde realizan el proceso de formación varían en intensidad. Las personas de ciencias básicas que pasan la mayor parte del tiempo juntas en el laboratorio establecen vínculos diferentes:

\begin{abstract}
... Sí, también cambian, porque uno se relaciona de una manera diferente... yo considero que uno es racional, entonces pues, se está en unas relaciones institucionales, personales o sentimentales o políticas e ideológicas que digamos. La universidad lo pone cuando lo traspone, están rehaciendo todo el tiempo, así como la representación social de la universidad afuera, así mismo uno empieza a representar a la universidad desde afuera, también depende de lo que uno estudie, si uno estudia Agronomía pues es más complicado, pero sí, si va a estudiar Sociología, Filosofía, Historia y ciertas cosas que tienen más que ver con lo humano (Álvaro, estudiante de Comunicación Social).
\end{abstract}

Independiente de la intensidad:

\begin{abstract}
... El hecho de sentirse parte de una comunidad académica le empieza a dar a uno más sentido de pertenencia, empieza a generarle visiones, entrar a un roce social con profesores, con personas que conocen muchas cosas y que le van a uno transmitiendo ideales, todo eso hace que uno se enfoque más y quiera presentar sus objetivos, sus metas (Alberto, estudiante de Administración de Empresas).
\end{abstract}

Los profesores interpretan dos roles distantes en la universidad y algo contradictorios. El primero, son protagonistas, representan la autoridad en el aula (ROCKWELL, 1997, p. 49), en las relaciones que se desarrollan en el proceso de formación son referentes; abren ventanas para tener contacto con sectores del conocimiento, dinámicas institucionales, particularidades de comunidades disciplinares (PIERELLA, 2009, p. 4) y sirven de puentes para interpretar sus mundos. Son artífices de las transformaciones que los estudiantes sufren durante su formación (YAIR, 2008, p. 96). El segundo rol, son actores de reparto, las reformas educativas han conducido a sacar a los profesores de su labor básica, cada vez es más frecuente que ocupen su tiempo en procesos administrativos, de certificaciones de calidad o inmersos en las relaciones o las pugnas por el poder dentro de las instituciones; el poder proveniente de procesos extraacadémicos cobra más fuerza en las instituciones. En especial, influyen en la experiencia estudiantil marginando a los profesores e imponiendo grupos de poder lejanos al ejercicio pedagógico como referentes para los estudiantes.

En Colombia, con la llegada de agentes privados a la educación secundaria y universitaria, los referentes cambiaron. Los representantes que observan los estudiantes institucionalmente son miembros de comunidades religiosas, grupos políticos o económicos, o los tres juntos. Establecen el vínculo entre el contexto y sus actuaciones, participan en la actualidad, en lo que sucede hoy en medios de comunicación; desbordan la universidad, tienen un rol protagónico en el contexto social, dejan una huella en los estudiantes y en el ejercicio docente. Al presentar a miembros de grupos de poder como políticos, predicadores o magnates, junto a un profesor, simplemente lo desplazan por los capitales simbólicos 
o económicos que poseen; estos empresarios son referentes para calificar la actividad de los profesores en las universidades privadas y también, cada vez más frecuente, en las públicas. El docente permanece en pugna constante entre ser protagonista o actor de reparto.

Hablando de la escuela primaria, Foglino et al. (2008, p. 238) perfilaron tres tipos de docentes, aproximados a las interpretaciones de los estudiantes universitarios. ${ }^{9}$ El tipo uno se relaciona con los profesores que se vinculan a la docencia como un peldaño para encontrar lugares estratégicos en el sistema educativo, en sectores administrativos, productivos privados o públicos. Algunos de estos profesores no están preparados o simplemente no tienen el tiempo suficiente para afrontar la docencia:

... Nosotros por ejemplo tenemos varios inconvenientes, yo ya he vivido varias
materias en donde hay profesores que uno dice "es en serio". Uno siente que llega
es a jugar al salón, llegan a ponerle a uno los trabajos que ellos tendrían que hacer
para sus trabajos, o como ponerse a pensar qué es lo que les voy a poner hacer
en esta clase, es terrible, (participantes del grupo de discusión de Arquitectura).

A esta tipología, miembros de grupo de discusión Administración de Empresas agregan:

... Con el pasar de los semestres, uno ve que los docentes, por un lado, no son como tan buenos, ni tan capacitados como deberían de ser para nuestro semestre, para nuestra carrera. Hemos visto que incluso en el primer semestre (no doy nombres) pero por la parte administrativa (de la universidad) delegan a algunos profesores para algunas materias y esos profesores realmente no son los adecuados para calificar las materias.

El primer tipo de docentes se relaciona con personas que no tienen la capacidad para establecer una relación fluida con los estudiantes, sus relaciones se basan en vinculaciones laborales. Su importancia para los jóvenes radica en que tienen empleos en un contexto con problemas económicos y, en especial, de desempleo; donde la obtención de un empleo digno y estable se convierte en un privilegio y en una preocupación de los estudiantes y de la mayoría de la población. Sus clases las orientan a partir de sus vivencias o trayectorias, presentadas como rutas que ellos pueden tomar, pueden ser "hacia la práctica" (PIERELLA, 2009, p. 16), a encontrar un empleo. Muchos han tenido inconvenientes con este tipo de profesores, pues las expectativas son altas y las realizaciones son bajas; así, el desarrollo de las clases se vuelve complejo, no existe interés por parte de los estudiantes de estar allí ni de los docentes.

El segundo tipo se relaciona con las personas que llegaron a la docencia sin tener interés en la actividad, ejercen el oficio pues no tienen o no alcanzan otras alternativas laborales. Son relaciones funcionales, preestablecidas, en las que solo buscan cumplir. La responsabilidad se descarga en los estudiantes:

9 Miembros del grupo de discusión de Licenciatura en Ciencia Naturales fueron más generosos y aumentaron el número de perfiles: “....dentro de la Universidad hay profesores buenos, regulares, malos y otros remalos, pues que no valoran bien al estudiante...” 
...Como lo venía diciendo el compañero, el profesor muchas veces entra, dicta la clase y sale y se va, no tiene como espacio para hablar y pone su nota, no está pendiente de usted de ¿cómo le fue?, en ese momento eso queda en otro plano, ya eso es como la responsabilidad de cada quien y si aprendió o no aprendió. El profesor no está midiendo si usted hizo el trabajo o por qué no lo hizo, sino, no lo hizo saca mala nota y si lo hizo pues bien, si sacó bien en el parcial pues bien y si no lo hizo el profesor no pregunta ¿bueno y por qué perdió el parcial? Entonces, eso es ya como la responsabilidad individual, el profesor no va a estar pendiente de por qué perdió (miembros del grupo de discusión en Licenciatura en Ciencias Naturales).

Adicional, este tipo de relaciones amplía el abismo generacional que hay entre los estudiantes y los profesores (BLANCO \& PIERELLA, 2009, p. 73); quienes van por obligación establecen un muro con los grupos que interactúan, desde luego cumple con sus obligaciones, pero no establece canales de comunicación y retroalimentación con los estudiantes. Viene el choque entre cumplir con la obligación y desarrollar el proceso de aprendizaje. Generalmente, la primera hace parte de la segunda. Sin embargo, asumir la docencia como un obligación hace que la calificación se convierta en el centro de las relaciones con los estudiantes, pues acorde con el tradicionalismo en educación, la evaluación es la mayor obligación del docente y el único mecanismo de medición; la retroalimentación constante de las actividades no se desarrolla; el proceso de aprendizaje y la interacción docente-estudiante pasa a segundo plano; el estudiante se responsabiliza de su propio proceso y hace lo que puede.

El último tipo, son quienes asumen la docencia como elección profesional y compromiso social. Estos profesores son destacados en los testimonios de los estudiantes, en ellos se evidencia la interacción constante entre docente y estudiante, son los modelos a seguir del estudiante y sus referentes para su proceso de formación:

Me gustaría resaltar el mérito de algunos profesores que están aquí por vocación, e impulsan el conocimiento extra a los que vienen interesados por la academia, resaltar y recuperar la academia como paso fundamental de la universidad. Es meritorio resaltar a todos ellos y a los estudiantes que no vienen por un cartón sino que buscan hacer algo más y no quedarse por la universidad (Participantes del grupo de discusión en Economía).

Dentro del Grupo de la Licenciatura en Lengua Castellana, una estudiante también quiso resaltar a uno de sus docentes:

... agregaría a una docente más, que me parece que es muy buena, ella ya no está acá, ahora está haciendo el doctorado, la profesora Érika..., me parece una excelente docente, ella trabaja en el área de Lingüística, ella es una lingüista total. Ella era la persona que uno llegaba a VII semestre y le decía a uno: no, es que usted no sabe escribir, uno que sí y ella que usted no sabe escribir, lo ponía a uno a escribir y le volvía una nada ese escrito. Yo creo que muchos estudiantes de la carrera se pasaron seis o siete semestres sin saber escribir, y más de uno se 
estrella con ella y decían que esa profesora es lo peor, es la que lo raja, es la que todo y mentiras, es una muy buena docente que lo ubicó a uno prácticamente, así como se dice, va por el camino equivocado, las cosas no son como usted cree y lo baja a uno de esa nube y lo pone a uno a pensar y a escribir, no perfectamente, pero sí de una forma muy diferente a como se venía escribiendo antes.

Julio (estudiante de Ingeniería Industrial), cuenta su experiencia con uno de los docentes que marcó su proceso:

Hay maneras (de aprobar la materia que dicta el profesor), por ejemplo, el de-
cano el semestre pasado, además toca tener una curva, una campana de Gauss,
para mirar a quién es que hay que arrastrar, eso de los que pasen, entonces, ahí
va pasando la gente y hay unos que enganchan, ahora la gente organiza estos
proyectos para evadir al profesor, hacen cursos vacacionales con otra persona
y hay formas de pasar... Ese man es complicadísimo, es un chiquitico, es una
porquería, pero bravo, a toda hora es bravo, pero ahi es en donde uno aprende,
yo creo que es la herramienta de primera mano que tenemos, lo que se aprende
en esa materia es demasiado.

Curiosamente, la tipología tres viene acompañada de la individualización del docente, sin importar qué tan difícil fue aprobar la materia, los estudiantes reconocieron el esfuerzo del docente por enseñarles y las implicaciones de ese profesional en su futuro. Es interesante observar cómo en diferentes carreras concuerdan en que los profesores que tienen capacidad profesional y compromiso académico son quienes establecen retroalimentación con los alumnos, una interacción constante guiada por un nivel de exigencia alto. Los profesores que se convierten en hitos dentro del proceso de formación, son con quienes aprenden, son con quienes los estudiantes debieron luchar y al final percibieron un cambio, una contribución a su formación.

Son tres tipos diferentes de profesores que identifican los estudiantes. El primero se relaciona con aquellos que encuentran lejos del aula su actividad principal, incluso afuera de la universidad; toman la docencia como una herramienta para complementar su actividad cotidiana. Su importancia radica en tener un empleo en una ciudad de pocas posibilidades laborales, esto los presenta frente a los estudiantes. El segundo tipo se refiere a los que entraron a la docencia por no encontrar otro espacio, se limitan a cumplir con sus obligaciones, en especial a cumplir con el desarrollo puntual de actividades, sin importar el proceso de formación que adelantan, incluso estableciendo una barrera entre ellos y los estudiantes. El último tipo, son quienes tienen compromiso con su labor, son referentes para el proceso de los estudiantes y su interacción se basa en la exigencia, son los profesores que marcan un hito y contribuyen en la formación de los estudiantes.

\section{A MANera de CIERRE}

Las ventanas son alternativas que encuentran los jóvenes en su cotidianidad para asumir su proceso de formación. El ideal es entrar, vivir, sufrir, disfrutar y terminar la 
universidad para tener una vida mejor. Reiteradamente, se dice a las personas que deben sobresalir laboral o académicamente en un contexto social conflictivo, con pocas oportunidades de superación económica y escasa movilidad social. Se presenta un choque entre lo planteado y lo desarrollado durante su proceso de formación; frente al resultado, las condiciones del entorno y las trayectorias de personas cercanas; todo influye significativamente en las elecciones o los caminos que transitan los estudiantes.

Tomar la formación universitaria como una ventana implica construir con formas de ver, actuar, sentir y pensar el mundo desde la universidad; es una esperanza para una vida $\mathrm{y}$ un futuro mejor. Los estudiantes encuentran los referentes para luchar en personas que observan en su realidad, Marina (estudiante de Biología) plantea:

Yo por el contrario pienso que ellos son el motivo por el cual la gente conti-
núa estudiando. Uno se da cuenta que esos que no siguieron estudiando... y
se pusieron a hacer otra cosa, pero están trabajando, no se la están ganando
fácil; pero de los que yo sé que no siguieron estudiando, la mayoría está mal,
ahí es cuando uno dice que vale la pena lo que estoy haciendo y vale la pena
continuar. Entonces, creo que es una afectación algo positiva, porque es como
un espejo en dónde mirarse uno y yo no quiero estar en esa situación... Así hay
mucha gente, incluidos mis compañeras (de colegio) que cada una tiene uno o
dos hijos, casi de la misma edad mía y con el marido que no les pega ni nada,
pero sin plata, ni nada, no se pueden ni moverse porque quién cuida los niños,
es complicado.

Reflexionando sobre su realidad, miran a sus compañeros, se dan cuenta que vale la pena seguir estudiando. Independiente de la pérdida de la importancia social de la educación superior, de los discursos que desmeritan la formación o que atacan a las universidades, los estudiantes o los docentes. Es un camino para enfrentar su particular situación.

A pesar de los discursos sobre tomar distancia o de los hitos que se promueven como alternativas a la formación superior, Alberto (estudiante de Administración de Empresas) sostiene:

Sin embargo, no es una excusa real, porque sí es difícil abrirse espacio en la vida con una formación académica, pues va a ser mucho más difícil sin esta formación. De manera que influye mucho, pero no tiene las bases como para que una teoría valga la pena realmente sostenerla, cada día el mundo es más exigente, por lo cual abrirse paso no es fácil.

Howard (estudiante de Ingeniería Industrial) agrega al respecto:

... Entonces, uno lo hace muchas veces es por facilitar el camino, o sea, es un camino mucho más fácil estudiando; porque se le facilitan las opciones de trabajo, puede clarificar qué es lo que busca, pues, no es una opción pero, siempre va a ser la de la educación, en eso sí estoy de acuerdo, va a ser mejor la de la educación. 
El hecho de pasar por la universidad brinda herramientas que la mayoría de los jóvenes no tienen para enfrentar una sociedad hostil.

Yolanda (estudiante en Licenciatura en Ciencias Naturales) continúa con la tensión entre camino fácil y la de luchar en la formación. Describe cómo prácticas cotidianas rechazables se pueden trasladar a los procesos de formación en la universidad, pero depende de cómo las personas las manejan y cómo asuman su trayectoria, su camino:

\begin{abstract}
Pues yo creo que pueden existir dos vías para eso. Una, el estudiante que ve al otro haciendo eso y dice, si él se lo consiguió así, yo por qué no. La otra como diría yo que puede ser mi caso, es que conseguir las cosas fácil, así como se consiguieron, así mismo se van; por ejemplo, algunos estudiantes pueden lograr pasar una nota, ofreciéndole cosas al profesor, haciendo fraude en las notas o cualquier cosa, no se siente la misma satisfacción cuando uno lo ha sudado para lograrlo, por lo menos decir que yo sé algo, un papel dice que yo sé algo. Pero que alguien me lo pregunte y que yo no sepa ni para dónde coger, porque no quiere decir que porque yo soy Licenciada en Ciencias Naturales, yo me las sé todas, con la educación que yo recibí puedo tener la facilidad de que si no sé algo, puedo... interpretarlo o asociarlo con algo, o saber dar una vía hacia una respuesta. Esos estudiantes que hacen fraude o que hacen cualquier otra cosa para conseguir las cosas, embarrada, porque el pobre más temprano que tarde va a caer.
\end{abstract}

Son las formas de asumir los procesos de formación. Es destacable el hecho que muchos estudiantes ven en su educación una herramienta que sobrepasa la necesidad de conseguir el título profesional; ellos buscan tener la capacidad desde su campo de interpretar y actuar sobre las cosas que pasan a su alrededor. Esta tipología de estudiante, usualmente, corresponde a aquellos que lucharon durante su formación y a pesar de tener vacíos, encuentran espacios académicos y senderos dónde generar alternativas para su entorno. El camino central es la lucha, involucrarse en un proceso de formación universitaria durante varios años, donde tienen que hacer esfuerzos y superar cotidianamente obstáculos; superar estas adversidades les brinda una ventana de vida diferente; son formas de asumir su rol en la sociedad, son formas de vincularse con mundos académicos o profesionales que parecieran distantes, son formas de desarrollarse individualmente y son formas de buscar alternativas de una vida mejor.

Los rasgos particulares de los estudiantes condicionan la relación, el desempeño y el desarrollo del proceso de formación. Estar en una comunidad donde se establecen condiciones para su pertenencia hace que la persona encuentre alternativas para el desarrollo de sus procesos de formación. El contexto, en especial, las condiciones de la carrera a la que pertenecen y las dinámicas de la comunidad académica, destacando los profesores, establecen las relaciones, los intercambios, las formas de actuar y asumir la formación. Desde luego, las capacidades se encuentran en este juego. Durante el proceso de formación el individuo va desarrollando capacidades gracias a su relación con la comunidad académica y las pone a su disposición para afrontar las adversidades o tomar decisiones. Los caminos que se les presentan a los estudiantes sirven como herramienta para enfrentar su proceso. 
Las condiciones de la población, el proceso educativo, las capacidades, los esfuerzos y los profesores, se encuentran en relación directa con los que ofrecen la comunidad académica particular y las alternativas que los estudiantes encuentran durante su formación.

\section{BibLIOGRAFÍA}

ACOSTA, F., CUBIDES, J. \& GALINDO, L. Sentidos y prácticas políticas en el mundo juvenil universitario. Bogotá: Universidad Nacional de Colombia, 2011.

BARRAGÁN, D. La experiencia estudiantil en una sociedad hostil. Una aproximación a los estudiantes universitarios de Ibagué (2012), (Tesis doctoral) Doctorado en Ciencias Sociales, Universidad de Buenos Aires (2017).

BARRAGÁN, D. \& PATIÑO, L. El trasfondo de las estadísticas. Elementos para la comprensión del fenómeno de la deserción universitaria en Colombia. Revista Latinoamericana de Administración, 9 (1), 55-66, 2013. Recuperado de: http://www.uelbosque.edu.

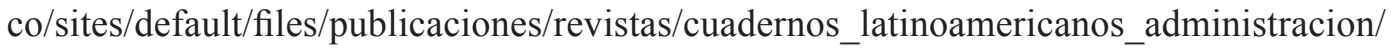
volumenIX_numero16_2013/desercion_universitaria.pdf.

BECKER, H., GEER, B., HUGHES, E. \& STRAUSS, A. Boys In White, Student culture in medical school. New Brunswisc: Transaction Publishers, 1992. Recuperado de: https:// es.scribd.com/doc/128463844/BECKER-Howard-S-Boys-in-White

BENAVIDES, A.; FRANCISCHETTO, V.; MARQUES, B.; MIRANDA, J.; NOGUEIRA, C.; LEME, V.; ARAÚJO, A \& ALMEIDA, L. O impacto das expectativas na adaptação acadêmica dos estudantes no Ensino Superior. Psico-USF, 19 (1), 49-60, 2014.

BLANCO, R. \& PIERELLA, M. Experiencias estudiantiles en la universidad contemporánea. Notas acerca de modos de abordaje de los discursos sobre autoridad, sexualidad y afectividad. Educación. Lenguaje y Sociedad, 6 (6), 69. México: Editorial ron, J.C. meBogot0)do de: s en una sociedad con profundos conflcitos y ia y numero de ad. vicnulacion en me-85, 2009. Recuperado de: http://www.biblioteca.unlpam.edu.ar/pubpdf/ieles/ n06a04blanco.pdf

BOURDIEU, P. Cuestiones de sociología. Madrid: Akal/Istmo, 2013.

CHÁVEZ, M. Ser indígena en la educación superior ¿desventajas reales o asignadas?. Revista de la Educación Superior, 37 (148), 31-55, 2008.

DUBET, F. Los estudiantes. Revista de Investigación Educativa, (1) 2-78, 2005. Recuperado de: http://www.uv.mx/cpue/num1/inves/completos/Dubet.pdf

DUBET, F. La experiencia sociológica. Barcelona: Gedisa, 2011.

ELÍAS, N. Mozart. Sociología de un genio. Barcelona: Península, 1991. 
ELÍAS, N. La sociedad de los individuos. Barcelona: Península, 2000.

ELÍAS, N. Sociología fundamental. Barcelona: Gedisa, 2011.

FOGLINO, A.; FALCONI, O. \& LÓPEZ, E. Una aproximación a la construcción de la experiencia escolar de adolescentes y jóvenes de grupos sociales urbanos en condiciones de pobreza en Córdoba. Cuadernos de Educación, 6 (6), 227-243, 2008. Recuperado de: http://www.revistas.unc.edu.ar/index.php/Cuadernos/article/viewFile/755/712

FRENCH, A. The experience of college undergraduates: degrees of transformation. (Phd Thesis) School of Management. Cranfield: Cranfield University, 1997. Recuperado de: https://dspace.lib.cranfield.ac.uk/handle/1826/3376

GOFFMAN, E. Frame Analysis. Los marcos de la experiencia. Madrid: CIS-Siglo XIX, 2006.

GOFFMAN, E. De cómo calmar al primo. Algunos aspectos de la adaptación al fracaso. Sociología Histórica, 2, 415-438, 2013.

GÓMEZ, M. \& ALZATE, M. El “oficio” de estudiante universitario: afiliación, aprendizaje y masificación de la Universidad. Pedagogía y Saberes, 33, 85-97, 2010.

GRAFF, G. Conflict pedagogy and student experience. College Composition and Communication, 46 (2), 276-279, 1995. Recuperado de: http://www.jstor.org/stable/358431

GUEVARA, H. Identidades estudiantiles, conocimiento y cultura. Percepciones de jóvenes universitarios y universitarias de Cuyo, Argentina. Revista Latinoamericana de Ciencias Sociales, Niñez y Juventud, 7 (1), 209-234, 2009.

KAPLAN, C. Buenos y malos alumnos: descripciones que predicen. Buenos Aires: Aique, 1994.

KAPLAN, C. Talentos, dones e inteligencias: el fracaso escolar no es un destino. Buenos Aires: Colihue, 2008.

MALINOWSKI, N. Diferenciación de los tiempos estudiantiles e impacto sobre el proceso de afiliación en México. Revista Latinoamericana de Ciencias Sociales, Niñez y Juventud, 6 (2), 801-819, 2008.

MÉNDEZ, A. Aristócratas del Saber. La formación de una elite igualitaria argentina en el Colegio Nacional de Buenos Aires. (Tesis doctoral) Doctorado en Ciencias Sociales, Universidad de Buenos Aires. Buenos Aires, 2010.

MINISTERIO DE EDUCACIÓN NACIONAL - MEN. Matrículas por departamento. Bogotá: Ministerio de Educación, (2012). Recuperado de: http://menweb.mineducacion. gov.co/seguimiento/estadisticas/principal.php?seccion=12\&id_categoria=1\&nivel=12\&d pto $=\&$ mun $=\&$ ins $=\&$ sede $=\&$ consulta_detalle $=$ total $\&$ consulta $=$ mat_total . 
NOBILE, M. \& ARROYO, M. Los efectos de experiencias escolarizadoras inclusivas sobre los relatos biográficos de docentes y estudiantes: un análisis de las Escuelas de Reingreso en Ciudad de Buenos Aires. RASE. Revista de la Asociación de Sociología de la Educación, 8 (3), 409-424, 2015.

PAOLONI, P. Trayectorias laborales y académicas. Puntos de encuentro y de desencuentro en estudiantes universitarios. Revista Iberoamericana de Educación, 55 (2), 1-11, 2011. Recuperado de: http://www.rieoei.org/deloslectores/3283Paoloni.pdf.

PIERELLA, M. Acerca de la autoridad de los profesores universitarios. Una aproximación a los discursos de estudiantes de la Universidad Nacional de Rosario en el tiempo presente. Ponencia. Universidad de Buenos Aires, Instituto Gino Germani, 2009. Recuperado de: http://webiigg.sociales.uba.ar/iigg/jovenes_investigadores/5jornadasjovenes/ EJE8/Maria\%20Paula\%20Pierella.pdf.

ROCKWELL, E. De huellas, bardas y veredas. Una historia cotidiana en la escuela. En: La escuela cotidiana. México: FCE, 1997.

ROLDAN, O. Incursión de los y las jóvenes en el mundo universitario: tensiones entre ser y permanecer. Educação \& Sociedade, 35 (126), 143-160, 2014. Recuperado de: https:// dx.doi.org/10.1590/S0101-73302014000100009.

SOLER, I.; ARIÑO, A. \& LLOPIS, R. Perfiles y regímenes de dedicación de los estudiantes universitarios. Univest, 9,1-13, 2009.

VÁZQUEZ, L. ¿Estudias y trabajas? Los estudiantes trabajadores de la Universidad Autónoma Metropolitana. Revista Latinoamericana de Estudios Educativos, 39 (3-4), 121149, 2009. Recuperado de: http://www.redalyc.org/pdf/270/27015078006.pdf.

YAIR, G. Key educational experiences and self-discovery in higher education. Teaching and Teacher Education, 24, 92-103, 2008.

YOUNG, M. EI triunfo de la meritocracia 1870-2034. Ensayos sobre educación y la igualdad. Madrid: Tecnos, 1963.

\section{Dados do autor:}

Diego Mauricio Barragán

Graduado em Contabilidade Pública. Mestre em Sociologia. Doutor em Ciências Sociais. Professor da Universidad Externado de Colombia.

Submetido em: 24-5-2017

Aceito em: 16-4-2018 